\title{
Treino de Memória Episódica com Ênfase em Categorização para Idosos sem Demência e Depressão
}

\author{
Episodic Memory Training with Emphasis on Categorization for Older Adults with- \\ out Dementia and Depression
}

\author{
Fabiana Castillo Roda Carvalho*, Anita Liberalesso Neri, \& Mônica Sanches Yassuda \\ Universidade Estadual de Campinas
}

\begin{abstract}
Resumo
Com o envelhecimento, podem ocorrer alterações cognitivas, especialmente, na memória. Ao mesmo tempo, as pesquisas apontam a possibilidade de melhorar a capacidade da memória por meio de treino cognitivo. O objetivo deste estudo foi verificar os efeitos do treino de memória episódica com idosos saudáveis brasileiros. Cinqüienta e sete idosos recrutados foram divididos aleatoriamente em grupo controle $(\mathrm{GCO}=26)$ e grupo experimental $(\mathrm{GEX}=31)$. Os participantes do grupo GEX participaram do pré-teste, seguido de cinco sessões de treino de memória episódica, nas quais foram instruídos a categorizar listas de supermercado e figuras, e do pós-teste. Os resultados indicaram que o treino de memória episódica envolvendo a aprendizagem e a prática com a estratégia de categorização promoveu melhora significativa no desempenho em tarefa de memória episódica e um maior uso da estratégia treinada.

Palavras-chave: Memória; Treino de memória; Idoso; Categorização.
\end{abstract}

\begin{abstract}
Aging may lead to cognitive changes, especially in memory. Yet, research suggests that older adults may improve memory performance after training. The present study aimed at evaluating the effects of episodic memory training offered to healthy Brazilian older adults. Fifty seven participants were randomly divided into control group $(\mathrm{COG}=26)$ and experimental group $(\mathrm{EXG}=31)$. The EXG participants completed a pre-test, five sessions of episodic memory training in which they were instructed to categorize grocery lists and pictures, and a post-test. Results indicated that episodic memory training involving learning and practice with categorization promoted significant improvement in episodic memory performance and in better using the trained strategy.

Keywords: Memory; Memory training; Older adults; Categorization.
\end{abstract}

O envelhecimento populacional é hoje uma realidade mundial, característica tanto dos países desenvolvidos como dos países em desenvolvimento. Durante o processo de envelhecimento, os idosos apresentam maior vulnerabilidade a doenças e uma parcela significativa é acometida por alterações cognitivas esperadas para a faixa etária. Essas alterações variam em sua forma e intensidade de indivíduo para indivíduo, devido a diferenças no estilo de vida, condições de saúde, alimentação, prática de atividades físicas, entre outros fatores (Newson \& Kemps, 2005).

Uma das alterações cognitivas que mais recebe atenção dos idosos é a de memória (Bertolucci, 2000). Salthouse (1991) afirma que, com o envelhecimento, ocorre uma redução na velocidade de processamento da informação que afeta o desempenho cognitivo, princi-

* Endereço para correspondência: Rua do Grito, 525, apto. 133, Ipiranga, São Paulo, SP, Brasil, CEP 04217-000. E-mail: fadrica_cr@uol.com.br palmente, em tarefas de memória e atenção. Alterações esperadas no envelhecimento incluem diminuição na memória operacional (Wood, Carvalho, Rothe-Neves, \& Haase, 2001) e na memória episódica (Charchat, Caramelli, Sameshima, \& Nitrini, 2005; Yassuda, Lasca, \& Neri, 2005).

Os estudos de treino de memória, por outro lado, demonstram que os idosos têm capacidade de aprender e empregar estratégias mnemônicas, tornando a gravação e a evocação de informações novas mais eficazes (Yassuda, Tavares-Batistoni, Fortes, \& Neri, 2006). Idosos aprendem a usar estratégias para as tarefas que lhes são ensinadas durante o treino, porém, não as generalizam para tarefas do seu cotidiano. Os estudos também informam que os efeitos do treino são mantidos, em geral, até seis meses, com algumas exceções na literatura, mostrando manutenção de maior duração (StigsdotterNeely \& Backman, 1993; Willis, Tennstedt, \& Marsiske, 2006). Desse modo, a literatura científica a respeito do treino de memória confirma a noção da plasticidade da memória no envelhecimento, mas sugere limitação na 
generalização e manutenção dos efeitos desses programas (Verhaeghen, Marcoen, \& Goossens, 1992).

Estudos mais recentes ilustram os avanços nas pesquisas sobre treino cognitivo para idosos. Ball et al. (2002), em estudo multicêntrico randomizado e controlado, que envolveu aproximadamente 2800 idosos entre 65 e 94 anos (com bom status cognitivo e independentes para realizar atividades de vida diária, AVDs), demonstrou que o treino cognitivo de 11 sessões pode gerar melhora significativa em uma parcela representativa de idosos que participa destas intervenções, particularmente, em habilidades como velocidade de processamento, raciocínio indutivo e memória. Entretanto, não foram detectadas alterações no desempenho nas atividades de vida diária, como preparar uma refeição, dirigir, fazer uso de medicação e administrar questões financeiras, talvez devido ao fato dos participantes não apresentarem declínio nesta esfera. Um estudo de segmento destes idosos (Willis et al., 2006) revelou uma boa manutenção dos ganhos associados ao treino inicial e menor declínio nas atividades de vida diária entre os participantes que receberam treino de raciocínio indutivo, sugerindo que o treino cognitivo pode ter implicações para a autonomia dos idosos.

Estudos brasileiros, como o de Lasca (2003), Wood et al. (2001), Yassuda et al. (2006), têm replicado os achados internacionais na população idosa brasileira, que apresenta menor escolaridade. Wood et al. (2000) ofereceram treino em velocidade de processamento para um grupo e no uso da técnica de associação para faces e nomes para outro grupo, cada grupo servindo de grupo controle para o outro para a técnica não treinada. Os grupos apresentaram melhor desempenho nas tarefas treinadas após quatro sessões, no entanto, os efeitos não se generalizaram para as tarefas não treinadas. Em outras palavras, o grupo que recebeu treino em velocidade de processamento não apresentou melhor desempenho na memorização de pares de faces e nomes.

Lasca (2003) ofereceu treino de categorização de sessão única para mulheres de 60 a 75 anos. O grupo experimental participou de um pré-teste, da sessão de treino e de um pós-teste realizado sete dias após o treino. O treino consistiu na oferta de instruções sobre como categorizar duas listas de itens de compras de supermercado, segundo critério de similaridade. No pós-teste, houve melhor desempenho na tarefa de memorização da lista de supermercado e maior uso da estratégia de categorização para o grupo experimental em comparação com o grupo controle, todavia, as diferenças não atingiram significância estatística.

Em estudo posterior, Yassuda et al. (2006) testaram a eficácia de treino de memória episódica de quatro sessões baseado em categorização para memorização de listas de supermercado e grifos para memorização de histórias. Resultados indicaram que, no pós-teste, o grupo experimental apresentou melhor desempenho na recordação de texto e maior uso de estratégias (organização da lista e uso de grifo). Os dois grupos (experimental e controle) relataram menor número de queixas em relação à memória e processamento de informações mais rápido no pós-teste.

O presente estudo objetivou oferecer um treino mais extenso enfatizando uma única estratégia mnemônica, visto que treinos com menor número de sessões, incluindo múltiplas estratégias, geraram efeitos modestos entre idosos brasileiros. Testou-se a hipótese de que, após o treino, os idosos apresentariam mudanças na memória episódica devido à plasticidade neuronal, fazendo maior uso da estratégia ensinada e apresentando maior velocidade de processamento.

\section{Métodos}

\section{Participantes}

$\mathrm{O}$ estudo foi realizado em um centro de convivência para a terceira idade. Foram recrutados 60 idosos que receberam esclarecimento sobre os objetivos e assinaram o Termo de Consentimento Livre e Esclarecido aprovado pelo comitê de ética local. Esses idosos foram distribuídos, aleatoriamente, em dois grupos controle (GCO), com $n=26$, e em dois grupos experimentais (GEX), com $n=31$. Nos grupos controle, 23 indivíduos eram do sexo feminino e 3 do sexo masculino, e nos grupos experimentais, 26 indivíduos eram do sexo feminino e 5 do sexo masculino. As comparações estatísticas realizadas entre o grupo controle e o grupo experimental (manhã e tarde juntos nas análises) revelaram que os grupos eram estatisticamente semelhantes quanto à idade, escolaridade, Escala de Depressão Geriátrica ([GDS], Paradela, Lourenço, \& Veras, 2005) e Mini-Exame do Estado Mental ([MEEM], Brucki, Nitrini, Caramelli, Bertolucci, \& Okamoto, 2003). Não foram incluídos no estudo idosos que apresentaram pontuação sugestiva de demência ou depressão no MEEM e na GDS, respectivamente. Duas idosas participantes não realizaram o pós-teste por motivo de doença, e uma idosa faltou mais de duas vezes nas sessões de treino e seus dados não foram incluídos nas análises. Assim, o total de idosos incluídos no estudo foi 57. A Tabela 1 apresenta os dados sócio-demográficos da amostra.

Tabela 1

Características Sócio-Demográficas da População Estudada em Médias e Desvios Padrão (entre parênteses), para Gênero são Relatados o Número de Participantes

\begin{tabular}{|c|c|c|}
\hline Gênero & $\begin{array}{c}\mathrm{GCO}(\mathrm{n}=26) \\
M(D P) \\
\mathrm{M}=23 \quad \mathrm{H}=3\end{array}$ & $\begin{array}{c}\text { GEX }(\mathrm{n}=31) \\
M(D P) \\
\mathrm{M}=26 \mathrm{H}=5\end{array}$ \\
\hline Idade & $70,04(4,39)$ & $69,10(5,31)$ \\
\hline Escolaridade & $4,85 \quad(1,91)$ & $5,32 \quad(2,75)$ \\
\hline GDS & $1,46 \quad(1,53)$ & $1,74 \quad(1,57)$ \\
\hline MEEM & $(2,17)$ & $26,90(2,56)$ \\
\hline
\end{tabular}

Nota. Não foram detectadas diferenças significativas entre os grupos. $\mathrm{M}=$ mulheres, $\mathrm{H}=$ homens. 


\section{Procedimentos}

A intervenção de memória oferecida aos grupos experimentais incluiu cinco sessões. Na primeira sessão, ocorreram o pré-teste e o início do treino de memória episódica, que continuou por mais quatro sessões. As sessões de treino do GEX ocorreram duas vezes por semana (às terças e quintas), cada uma com duração de 60 minutos. Na sexta sessão, foi realizado o pós-teste. Os grupos GCO realizaram o pré-teste e o pós-teste nas mesmas semanas dos grupos GEX e receberam uma versão abreviada do treinamento na semana após as avaliações de pós-teste. As sessões foram desenvolvidas em grupos de 13 a 23 pessoas.

Durante as cinco sessões de treino, os participantes foram ensinados a utilizar a estratégia de categorização. Foi apresentado o conceito de categorização em todas as sessões e treinado o uso da técnica por intermédio de material visual ou verbal, alternadamente.

O treino iniciava a sessão com a distribuição do material visual ou verbal, trabalhado em casa. A pesquisadora sugeria que os participantes dividissem os itens em categorias em aproximadamente 20 minutos, individualmente. Depois disso, a pesquisadora realizava a mesma tarefa em grupo com o mesmo material e, em seguida, os participantes tentavam recordar os itens estudados. A seguir, realizava-se o exercício de categorização com a modalidade não realizada em casa nem no primeiro exercício. Como exercício de casa, os participantes levavam um material visual ou verbal similar ao usado no segundo exercício da sessão. Por exemplo, se a tarefa de casa envolvesse material verbal, o primeiro exercício da sessão utilizaria este material, e o segundo exercício, assim como a tarefa de casa da próxima sessão, utilizariam material visual. Os materiais visuais utilizados consistiram de quatro pranchas de figuras coloridas extraídas do teste SKT, Short Cognitive Performance Test, já validado junto à população brasileira (Erzigkeit, 2001; Flaks et al., 2006). Cada prancha continha 12 figuras, e era possível categorizá-las em sub-grupos como animais, objetos de cozinha, entre outros.

Para os materiais verbais foram utilizadas quatro listas de supermercado (Yassuda et al., 2005), com 35 itens em cada uma delas. Estes itens podiam ser categorizados em sete sub-grupos (tipos de produtos que podem ser adquiridos em supermercado), como materiais de limpeza, frutas, verduras, entre outros.

A adesão às tarefas de casa foi muito alta e, raramente, um participante deixava de fazer a tarefa solicitada. Não foi observada diferença entre os grupos quanto à realização das tarefas. Entretanto, não foi realizado um controle objetivo da freqüência de realização das tarefas de casa.

Os participantes do grupo controle receberam uma sessão de treino cognitivo de 120 minutos, durante a qual receberam informações sobre envelhecimento cognitivo e instruções sobre a estratégia de categorização.

\section{Materiais}

Medida de Memória Episódica. Para a avaliação da memória episódica foram utilizadas duas pranchas com
18 figuras (versão A e B), que podiam ser divididas em três categorias com seis itens em cada uma. As figuras das pranchas foram extraídas do trabalho de Pompéia, Miranda e Bueno (2001), que forneceram dados normativos para o Brasil de 400 figuras de objetos avaliados por estudantes universitários e crianças de cinco a sete anos. No referido estudo, foram avaliadas a consistência na nomeação, a familiaridade com os objetos e a complexidade visual dos desenhos. Esses dados possibilitaram a utilização desse conjunto de figuras como ferramenta para pesquisas cognitivas. As 18 figuras escolhidas para compor as pranchas A e B apresentavam bons índices de nomeação, familiaridade e complexidade. A adequação e a equivalência das duas versões das pranchas de figuras foram testadas em testes piloto (dados disponíveis se solicitados aos autores) anteriores à realização do estudo.

Nessa tarefa, os participantes tinham um minuto para estudar a prancha e, após a realização da prova Códigos do WAIS-R em 90 segundos (Wechsler, 1981), eram solicitados a escrever os nomes das figuras que conseguissem lembrar. Optou-se por usar 90 segundos para a realização da prova Códigos, pois testagens piloto indicaram que este intervalo de tempo evitava o efeito de teto e de solo no resgate das figuras memorizadas.

As versões A e B das pranchas foram usadas simultaneamente nas sessões de testagem, entre os múltiplos grupos de pré e pós-teste, para não ficarem atreladas a nenhuma condição experimental ou ao tempo de teste.

Velocidade de Processamento. Para examinar possíveis mecanismos que pudessem explicar os efeitos positivos da intervenção, além do uso de estratégias, avaliou-se a velocidade de processamento cognitivo dos participantes. Para avaliar a velocidade de processamento de informações dos participantes, usou-se o subteste de transferência de símbolos (Códigos) da bateria de inteligência WAIS-R (Wechsler, 1981), utilizada como uma medida de velocidade de processamento em diversas pesquisas anteriores.

Nessa tarefa, os participantes tinham que preencher os quadrados em branco com o símbolo pareado com cada número, o mais rápido possível dentro de 90 segundos. A variável de interesse foi a quantidade de símbolos que conseguiram transferir nesse limite de tempo.

Avaliação do Uso de Estratégia Através de Auto-Relato. Após a realização da tarefa de memória, os participantes tinham que responder, por meio de pergunta aberta, quais estratégias teriam utilizado para memorizar as figuras. Em seguida, foi oferecida uma lista com estratégias possíveis para os participantes indicarem se haviam usado ou não tais estratégias.

Uso de Estratégia Direto no Protocolo. O uso de estratégia também foi avaliado diretamente no protocolo do pré e do pós-teste. Na memorização das figuras, foi calculada a medida de categorização de Bousfield (1953), utilizada frequientemente em estudos de memorização de listas (West \& Thorn, 2001; no Brasil ver Mello, 2003). Para a obtenção desse índice, calcula-se a taxa de repeti- 
ção intra-categórica (ratio of repetition), seguindo a fórmula $[\mathrm{RR}=r / n-1]$, onde $\mathrm{r}$ representa o número de itens de uma mesma categoria recordados juntos e $\mathrm{n}$ o total de itens recordados da lista. Esse índice varia de 0 (nenhuma categorização) a 1 (categorização total).

\section{Resultados}

Para comparação das variáveis categóricas entre os grupos, foi utilizado o teste Qui-Quadrado ou, quando necessário (poucos participantes por célula), o teste exato de Fisher. Diversas variáveis não apresentaram distribuição normal, assim, testes não paramétricos foram utilizados. Para comparação das variáveis contínuas entre grupos, foi utilizado o teste de Mann-Whitney. Para comparação dos tipos de estratégias entre os tempos em cada grupo, foi utilizado o teste de Mc Nemar. Para analisar a relação entre as variáveis numéricas de interesse, foi calculado o coeficiente de correlação de Spearman. Na comparação da evolução dos parâmetros entre os grupos e tempos de coleta, foi utilizada a Análise de Variância para medidas repetidas. Para comparar os escores entre os tempos, foi utilizado o teste de perfil por contrastes e, para comparar os escores entre os grupos, foi utilizado o teste de Tukey. O nível de significância adotado para os testes estatísticos foi de $5 \%(p<0,05)$. O tratamento estatístico foi apoiado pelos programas computacionais SAS (System for Windows) 8.02.

Os resultados da medida de memória episódica dos grupos GCO e GEX estão descritos na Tabela 2. Análises realizadas com o teste de Mann-Whitney revelaram diferença significativa entre o pré e pós-teste somente para o grupo experimental. Essas análises também revelaram que os grupos GCO e GEX apresentaram desempenho estatisticamente equivalente no pré-teste.

A análise dos valores mínimos e máximos para o desempenho na tarefa de memória indicou que não houve efeito de teto, isto é, desempenho máximo, para nenhum dos grupos em nenhum dos tempos de avaliação. No préteste, o desempenho mínimo foi de 4 itens e o máximo de 15 itens para ambos os grupos. No pós-teste, o desempenho mínimo foi de 5 itens e máximo de 17 itens para o GCO, e 6 e 16 para o GEX, respectivamente.

Tabela 2

Média e Desvio Padrão (entre parênteses) para a Medida de Memória Episódica

\begin{tabular}{lcc} 
& Pré-teste & Pós-teste \\
& $M(D P)$ & $M(D P)$ \\
\hline GCO & $8,85(3,21)$ & $10,27(2,75)$ \\
GEX & $8,45(2,57)$ & $11,94^{\mathrm{a}}(3,05)$ \\
\hline
\end{tabular}

Nota. ${ }^{\text {a }}=$ diferença entre Pré e Pós para os GEXs atingiu significância estatística em $p<0,05$.

Os resultados da medida de velocidade de processamento na tarefa Códigos dos grupos GCO e GEX, no pré e pós-teste, estão descritos na Tabela 3. Análises das médias dos grupos nos dois tempos demonstraram que a velocidade de processamento permaneceu inalterada entre os dois tempos de avaliação para os dois grupos, sugerindo que o treino e a prática oferecida pela retestagem não aumentaram a velocidade de processamento dos participantes.

Tabela 3

Média e Desvio Padrão (entre parênteses) para Códigos (WAIS-III)

\begin{tabular}{lcc}
\hline & Pré-teste & $\begin{array}{c}\text { Pós-teste } \\
M(D P)\end{array}$ \\
\hline GCO & $21,73(8,49)$ & $22,48(9,20)$ \\
GEX & $27,31(10,54)$ & $27,55(8,77)$ \\
\hline
\end{tabular}

A medida de categorização de Bousfield dos grupos GCO e GEX, nas duas avaliações, está descrita na Tabela 4. Análises demonstraram que houve diferença significativa na intensidade da categorização entre o pré e o pós-teste somente para os grupos GEX. Os grupos GCO não apresentaram aumento significativo na categorização dos itens durante o resgate da informação entre as avaliações.

Tabela 4

Média e Desvio Padrão (entre parênteses) para a Medida de Bousfield

\begin{tabular}{lcc}
\hline & $\begin{array}{c}\text { Pré-teste } \\
M(D P)\end{array}$ & \multicolumn{2}{c}{ Pós-teste } \\
& \multicolumn{2}{c}{$M(D P)$} \\
\hline GCO & $0,43(0,21)$ & $0,47 \quad(0,22)$ \\
GEX & $0,42(0,20)$ & $0,63^{\mathrm{a}}(0,20)$ \\
\hline
\end{tabular}

Nota. ${ }^{\text {a }}=$ diferença entre o Pré e o Pós-teste para o GEX atingiu significância estatística em $p<0,05$.

A Tabela 5 apresenta os resultados das ANOVAs para medidas repetidas com o intuito de comparar os parâmetros entre os grupos e os tempos de coleta. Observando os resultados da Tabela 5, verifica-se interação significativa entre os fatores Grupo e Tempo para as variáveis de desempenho de memória episódica e para a medida de categorização de Bousfield. Para estas duas variáveis, houve também um efeito significativo do fator Tempo. Análise das médias revelou que houve diferença significativa entre os grupos GEX e o GCO no pós-teste e diferença significativa entre pré e pós-teste somente para o grupo experimental. Estes resultados sugerem que os grupos GEX apresentaram maior ganho em desempenho de memória e uso da categorização após o treino do que os GCO. As análises também revelaram efeito significativo do fator Tempo para as variáveis Códigos e Número de estratégias usadas. Análise das médias revelou que tanto os grupos GEX como os GCO aumentaram sua velocidade de processamento e o número de estratégias usadas. 
Carvalho, F. C. R., Neri, A. L. \& Yassuda, M. S. (2010). Treino de Memória Episódica com Ênfase em Categorização para Idosos sem Demência e Depressão.

Tabela 5

Resultados das ANOVAs para Comparação dos Escores entre Grupos e Tempos

\begin{tabular}{|c|c|c|c|}
\hline Parâmetros & $\begin{array}{c}\text { Comparação entre } \\
\text { Grupos (GC vs GE) }\end{array}$ & $\begin{array}{l}\text { Comparação entre } \\
\text { Tempos (Pré e Pós) }\end{array}$ & $\begin{array}{c}\text { Efeito da Interação } \\
\text { Grupo vs Tempo }\end{array}$ \\
\hline Códigos & $F_{(1,55)}=0,04 ; p=0,835$ & $F_{(1.55)}=71,36 ; p<0,001^{a}$ & $F_{(155)}=0,17 ; p=0,686$ \\
\hline Memória - Recordação & $F_{(1,55)}^{(1, J))}=1,06 ; p=0,308$ & $F_{(1,55)}^{(1,0 \mathcal{)})}=28,28 ; p<0,001^{\mathrm{b}}$ & $F_{(1,55)}=4,99 ; p=0,030^{\mathrm{b}}$ \\
\hline Medida de Bousfield & $F_{(1,55)}^{(1,03)}=3,05 ; p=0,087$ & $F_{(155)}^{(1,5 \mathcal{J})}=13,05 ; p<0,001 \mathrm{~b}$ & $F_{(1,55)}^{(1, \mathcal{O})}=6,89 ; p=0,011^{\mathrm{b}}$ \\
\hline Número de Estratégias Usadas & $F_{(1,55)}^{(1,5)}=0,83 ; p=0,367$ & $F_{(1,55)}^{(1,3))}=17,56 ; p<0,001^{\mathrm{a}}$ & $F_{(1,55)}=3,26 ; p=0,076$ \\
\hline
\end{tabular}

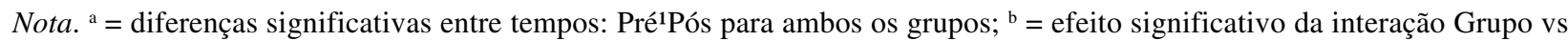
Tempo: diferença significativa entre grupos no tempo Pós (GCOGEX); e diferença significativa entre tempos apenas para o GEX (Pré1Pós).

Quanto ao uso de estratégias, dados obtidos pela pergunta aberta geraram respostas genéricas como "Nada", "Pensei", "Memorizei", "Lembrei". Não foram observados nomes de estratégias. Portanto, estes dados não foram analisados quantitativamente, mas sim qualitativamente, e sugerem que estes participantes tinham dificuldade em reconhecer ou nomear possíveis estratégias utilizadas.

A Tabela 6 apresenta o número bruto de pessoas que relatou ter usado uma determinada estratégia na questão fechada que oferecia uma lista de possíveis estratégias. Entre parênteses, encontra-se a porcentagem de pessoas que indicaram ter usado a estratégia. Análises estatísticas realizadas com o teste Qui-quadrado e o teste Exato de Fisher demonstraram que os grupos GCO e GEX, no pré-teste, usaram espontaneamente, com maior frequiência, a estratégia de imagem mental, enquanto que, no pós-teste, os grupos GCO diminuíram o uso da estratégia de imagem mental e os GEX mantiveram a freqüência do uso da imagem mental. Para a estratégia de organização em grupos semânticos, as análises indicaram que ambos os grupos aumentaram o uso desta estratégia, contudo, o aumento foi de maior magnitude nos grupos GEX. Para as outras estratégias apresentadas na tabela, não foram encontradas diferenças entre os grupos nos dois momentos de testagem.

As análises de correlação de Spearman revelaram que, no pós-teste, os indivíduos dos dois grupos que foram mais rápidos tiveram maior número de itens recordados e os indivíduos que mais usaram a estratégia treinada (medida de categorização de Bousfield) tiveram melhor desempenho na recordação dos itens. Porém, houve associação mais forte entre a medida de Bousfield e a tarefa de memória para os GEX. Estes resultados não estão apresentados devido à limitação de espaço.

\section{Discussão}

Estudos anteriores (Willis et al., 2006; Yassuda et al., 2006) sugeriram que o treino de memória pode promover mudanças no funcionamento cognitivo em idosos e colaborar para manter sua funcionalidade e independência. Os estudos de treino de memória no envelhecimento normal são escassos no Brasil, isto é, não há dados suficientes para confirmar se os achados dos estudos de treino de memória com populações internacionais são aplicáveis para a nossa população devido às características sócio-demográficas diferentes.

Baseado em pesquisas internacionais e brasileiras, o presente estudo encontrou alterações estatisticamente significativas para o desempenho de memória episódica e para o uso da categorização após treino mnemônico de cinco sessões. Estes ganhos foram superiores ao efeito da retestagem apresentado pelo grupo controle.

Os resultados positivos do treino de memória episódica podem estar relacionados a alguns fatores. O primeiro refere-se ao treino unifatorial que foi oferecido. Isto é, foi ensinado somente um tipo de estratégia, a categorização. Levando-se em consideração que a população envolvida tinha em média quatro anos de escolaridade, é possível que o treino intensivo com estratégia única seja o mais eficaz, visto que gerou resultados de maior magnitude que a intervenção anterior de quatro sessões com ênfase em duas estratégias (Yassuda et al., 2006). Estudos multifatorais extensos também geraram resultados significativos, entretanto, envolveram população mais escolarizada e tiveram maior duração, acima de 10 sessões (Stigsdotter-Nelly \& Backman, 1993).

Um segundo fator que poderia estar associado à melhora do desempenho está relacionado à intensidade do treino. Wood et al. (2000) apontaram que os idosos com quatro a oito anos de escolaridade apresentam melhor desempenho a partir da quarta sessão de treino. Dados do presente estudo estão em congruência com estes achados. Estudo anterior de Lasca (2003), envolvendo a estratégia de categorização para a memorização de listas de supermercado, indicou uma modesta melhora após uma única sessão de treino. Estudos anteriores sugeriram que treinos curtos podem levar à deficiência da utilização, isto é, ao uso da estratégia mnemônica sem benefício no desempenho (Lasca, 2003; Yassuda et al., 2006).

Em congruência com a literatura internacional (CaleroGarcía \& Navarro-González, 2007), os resultados deste estudo revelaram uma correlação significativa entre os escores de memória episódica e o uso de categorização no pós-teste para ambos os grupos, sendo essa associa- 
Tabela 6

Uso de Estratégia por Meio do Auto-Relato, Número de Pessoas que Indicaram ter Usado a Estratégia e entre parênteses a Porcentagem de Pessoas

\begin{tabular}{|c|c|c|}
\hline Grupo & Pré-teste & Pós-teste \\
\hline & Imagem Menta & \\
\hline \multicolumn{3}{|l|}{ Controle } \\
\hline Não & $4 \quad(15,38)$ & $10(38,46)^{\mathrm{a}}$ \\
\hline Sim & $22(84,62)$ & $16(61,54)$ \\
\hline \multicolumn{3}{|c|}{ Experimental } \\
\hline Não & $4 \quad(12,90)$ & $4(12,90)$ \\
\hline \multirow[t]{2}{*}{ Sim } & $27(87,10)$ & $27(87,10)^{\mathrm{a}}$ \\
\hline & Organização em Gr & \\
\hline \multicolumn{3}{|l|}{ Controle } \\
\hline Não & $18(69,23) 13$ & $(50,00)^{\mathrm{a}}$ \\
\hline Sim & $8(30,77)$ & $13(50,00)$ \\
\hline \multicolumn{3}{|c|}{ Experimental } \\
\hline Não & $21(67,74)$ & $7 \quad(22,58)$ \\
\hline \multirow[t]{2}{*}{ Sim } & $10(32,26)$ & $24(77,42)^{\mathrm{a}}$ \\
\hline & Associação & \\
\hline \multicolumn{3}{|l|}{ Controle } \\
\hline Não & $20(76,92)$ & $13 \quad(50,00)$ \\
\hline Sim & $6(23,08)$ & $13 \quad(50,00)$ \\
\hline \multicolumn{3}{|c|}{ Experimental } \\
\hline Não & $20(64,52)$ & $15 \quad(48,39)$ \\
\hline \multirow[t]{2}{*}{ Sim } & $11(35,48)$ & $16 \quad(51,61)$ \\
\hline & Primeiras Letra & \\
\hline \multicolumn{3}{|l|}{ Controle } \\
\hline Não & $22(84,62)$ & $24 \quad(92,31)$ \\
\hline Sim & $4(15,38)$ & $2(7,69)$ \\
\hline \multicolumn{3}{|c|}{ Experimental } \\
\hline Não & $28(90,32)$ & $25 \quad(80,65)$ \\
\hline \multirow[t]{2}{*}{ Sim } & $3(9,68)$ & $6 \quad(19,35)$ \\
\hline & Pensou em objetos que & \\
\hline \multicolumn{3}{|l|}{ Controle } \\
\hline Não & $16(61,54)$ & $14 \quad(53,85)$ \\
\hline Sim & $10(38,46)$ & $12(46,15)$ \\
\hline \multicolumn{3}{|c|}{ Experimental } \\
\hline Não & $20(64,52)$ & $17 \quad(54,84)$ \\
\hline \multirow[t]{2}{*}{ Sim } & $11(35,48)$ & $14(45,16)$ \\
\hline & Contou quantas figuras havia no tota & \\
\hline \multicolumn{3}{|c|}{ 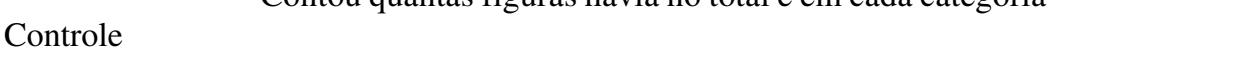 } \\
\hline Não & $21(80,77)$ & $20 \quad(76,92)$ \\
\hline Sim & $5(19,23)$ & $6 \quad(23,08)$ \\
\hline \multicolumn{3}{|c|}{ Experimental } \\
\hline Não & $27(87,10)$ & $24 \quad(77,42)$ \\
\hline \multirow[t]{2}{*}{ Sim } & $4(12,90)$ & $7 \quad(22,58)$ \\
\hline & Ordem em que as figuras & \\
\hline \multicolumn{3}{|c|}{ 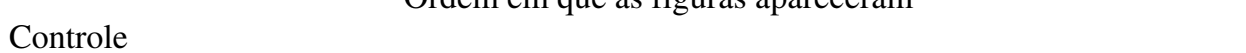 } \\
\hline Não & $21(80,77)$ & $23 \quad(88,46)$ \\
\hline Sim & $5(19,23)$ & $3(11,54)$ \\
\hline \multicolumn{3}{|c|}{ Experimental } \\
\hline Não & $27(87,10)$ & $22 \quad(70,97)$ \\
\hline Sim & $4(12,90)$ & $9 \quad(29,03)$ \\
\hline
\end{tabular}

Nota . Não = não usou a estratégia Sim = usou a estratégia $;$ GCO = grupo controle; GEX = grupo experimental; ${ }^{\mathrm{a}}=$ diferença entre o GCO e o GEX atingiu significância estatística em $p<0,05$. 
ção mais intensa para o grupo experimental. Esses dados sugerem que o uso de estratégias mnemônicas pode favorecer a memorização e que o treino pode intensificar a associação entre desempenho e uso de estratégia.

Intervenções de memória, com o objetivo de melhorar o desempenho em tarefas mnemônicas são importantes para a funcionalidade do idoso, como lembrar-se de tomar remédios, de pagar as contas, de preparar refeições balanceadas, e podem contribuir para a independência do idoso e diminuir riscos de institucionalização. Devese ressaltar, todavia, que estudos de treino de memória com ênfase na memória prospectiva (lembrar de lembrar), tão relevante para a funcionalidade do idoso, são ainda raros na literatura.

Uma das limitações deste estudo foi não ter avaliado a generalização dos efeitos do treino para as tarefas do diaa-dia. Outra limitação refere-se a não inclusão de medidas de manutenção dos efeitos do treino por meio de reavaliações após determinados períodos.

Deve-se ressaltar a importância do tema referente às intervenções cognitivas para o campo da Gerontologia, visto que o bom funcionamento cognitivo está diretamente relacionado à autonomia do idoso. Futuras pesquisas deveriam superar as limitações do presente estudo, com a inclusão de investigações sobre treino cognitivo multifatorial, com avaliações de seguimento e de transferência das estratégias para o cotidiano, uma vez que esta linha de pesquisa, no Brasil, está em fase inicial.

\section{Referências}

Ball, K., Berch, D. B., Helmers, K. F., Jobe, J. B., Leveck, M. D., Marsiske, M., et al. (2002). Effects of cognitive training interventions with older adults: A randomized controlled trial. Journal of the American Medical Association, 288(18), 2271-2281.

Bertolucci, P. (2000). Instrumentos para rastreio das demências. In O. V. Forlenza (Ed.), Neuropsiquiatria Geriátrica (pp. 65-80). São Paulo, SP: Atheneu.

Bousfield, W. A. (1953). The occurrence of clustering in the recall of randomly arranged associates. Journal of General Psychology, 49, 229-240.

Brucki, S. M. D., Nitrini, P., Caramelli, P., Bertolucci, P. H. F., \& Okamoto, I. H. (2003). Sugestões para o uso do MiniExame do Estado Mental no Brasil. Arquivos de Neuropsiquiatria, 61(3-B), 777-781.

Calero-García, M. D., \& Navarro-González, E. (2007). Effectiveness of a memory training programme in the maintenance of mental status in elderly people with and without cognitive decline. Psychology in Spain, 11(1), 107112.

Charchat, H., Caramelli, P., Sameshima, K., \& Nitrini, R. (2005). Declínio da capacidade cognitiva durante o envelhecimento. Revista Brasileira de Psiquiatria, 27(12), 79-82.

Erzigkeit, H. (2001). A Short Cognitive Performace Test for assessing deficits for memory and attention - SKT. Erlangen, Germany: Geromed.

Flaks, M. K., Yassuda, M. S., Regina, A. C. B., Cid, C. G., Camargo, C. H. P., Gattaz, W. F., et al. (2006). The Short
Cognitive Performance Test (SKT): A preliminary study of its psychometric properties in Brazil. International Psychogeriatrics, 18, 121-133.

Lasca, V. B. (2003). Treinamento de memória no envelhecimento normal: Efeitos de um programa aplicado a idosos. Dissertação de Mestrado não-publicada, Faculdade de Educação, Universidade Estadual de Campinas, SP.

Mello, C. B. (2003). Estratégias categóricas de recordação e formação de conceitos em crianças de sete a quatorze anos de idade. Tese de Doutorado não-publicada, Universidade de São Paulo, SP.

Newson, R. S., \& Kemps, E. B. (2005). General lifestyle activities as a predictor current cognition and cognitive change in older adults: A cross-sectional and longitudinal examination. The Journals of Gerontology, 60(3), 113-20.

Paradela, E. M. P., Lourenço, R. A, \& Veras, R. P. (2005). Validação da Escala de Depressão Geriátrica em um ambulatório geral. Revista Saúde Pública, 39(6), 918-923.

Pompéia, S., Miranda, M. C., \& Bueno, O. F. A. (2001). A set of 400 pictures standardised for portuguese. Arquivos de Neuropsiquiatria, 59(2-B), 330-337.

Salthouse, T. A. (1991). Theoretical perspectives on cognitive aging. Hillsdale, NJ: Lawrence Erlbaum.

Stigsdotter-Neely, A., \& Backman, L. (1993). Long-term maintenance of gains from memory training in older adults: Two 3.5-year follow-up studies. Journal of Gerontology, 48(5), 233-237.

Verhaeghen, P., Marcoen, A., \& Goossens, L. (1992). Improving memory performance in the aged trough mnemonic training: A meta-analytic study. Psychology and Aging, 7(2), 242-251.

Wechsler, D. I. (1981). Examiner's Manual: Wechsler Adult Intelligence Scale-Revised. New York: Psychological Corporation.

West, R. L., \& Thorn, R. M. (2001). Goal-setting, self efficacy, and memory performance in older and younger adults. $E x$ perimental Aging Research, 27, 41-65.

Willis, S. L., Tennstedt, S. L., \& Marsiske, M. (2006). Longterm effects of cognitive training on everyday functional outcomes in older adults. Journal of the American Medical Association, 269(23), 2805-2814

Wood, G. M. O., Carvalho, M. R. S., Rothe-Neves, R., \& Haase, V. G. (2001). Validação da Bateria de Avaliação da Memória de Trabalho (BAMT-UFMG). Psicologia: Reflexão $e$ Crítica, 14(2), 325-341.

Wood, G. M. O., Haase, V. G., Araujo, J. R., Scalioni, I. G., Lima, E. P., \& Sampaio, J. R. (2000). Desenvolvimento cognitivo adulto: A avaliação e a reabilitação da capacidade de memória de trabalho. In G. M. O Wood (Ed.), Psicologia do Desenvolvimento: Contribuições interdisciplinares (pp. 121-144). Belo Horizonte. MG: Health.

Yassuda, M. S, Lasca, V. B., \& Neri, A. L. (2005). Meta-memória e auto-eficácia de instrumentos de pesquisa. Psicologia: Reflexão e Crítica, 18(1), 78-90.

Yassuda, M. S., Tavares-Batistoni, S. S., Fortes, A. G., \& Neri, A. L. (2006). Treino de memória no idoso saudável: Benefícios e mecanismos. Psicologia: Reflexão e Crítica, 19(3), 470-481. 